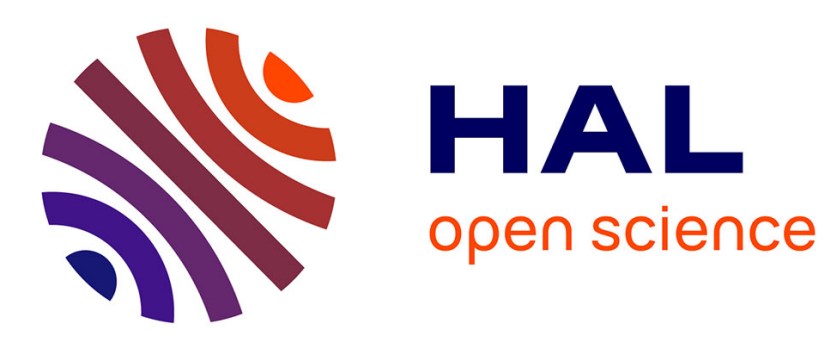

\title{
SDR for SRD: ADC specifications for reconfigurable gateways in urban sensor networks
}

Mathieu Vallerian, Guillaume Villemaud, Benoit Miscopein, Tanguy Risset, Florin Hutu

\section{- To cite this version:}

Mathieu Vallerian, Guillaume Villemaud, Benoit Miscopein, Tanguy Risset, Florin Hutu. SDR for SRD: ADC specifications for reconfigurable gateways in urban sensor networks. IEEE Radio Wireless Symposium, Jan 2014, Newport Beach, United States. hal-00920906

\section{HAL Id: hal-00920906 https://hal.inria.fr/hal-00920906}

Submitted on 19 Dec 2013

HAL is a multi-disciplinary open access archive for the deposit and dissemination of scientific research documents, whether they are published or not. The documents may come from teaching and research institutions in France or abroad, or from public or private research centers.
L'archive ouverte pluridisciplinaire HAL, est destinée au dépôt et à la diffusion de documents scientifiques de niveau recherche, publiés ou non, émanant des établissements d'enseignement et de recherche français ou étrangers, des laboratoires publics ou privés. 


\title{
SDR for SRD: ADC specifications for reconfigurable gateways in urban sensor networks
}

\author{
Mathieu Vallérian ${ }^{1,2}$, Guillaume Villemaud ${ }^{2}$, Benoit Miscopein ${ }^{1}$, Tanguy Risset ${ }^{2}$, and Florin Hutu ${ }^{2}$ \\ ${ }^{1}$ Orange-Labs, 38243, Meylan , France, \\ ${ }^{2}$ Université de Lyon, INRIA, INSA-Lyon, CITI-INRIA, F-69621, Villeurbanne, France
}

\begin{abstract}
Short Range Devices (SRD) are increasingly employed in urban sensor networks using different communication protocols. That becomes a key problem in the gateway design, since its cost and energy consumption increase with the number of implemented technologies. This cost and energy can be reduced by using a reconfigurable gateway to perform the biggest part of signal processing digitally, as it is done in Software-Defined Radio (SDR).

As several received signals should be simultaneously digitized, Analog-to-Digital Converter (ADC) must be able to treat the whole frequency band used by SRD, with a high enough resolution to properly demodulate the signals. This paper describes what the ADC's constraints are and how to dimension an ADC in SDR for SRD. An example based on the SmartSantander deployment shows that an ADC resolution of 23 bits is required to properly demodulate the data.

Index Terms-Analog-digital conversion, software radio, wireless sensor networks
\end{abstract}

\section{INTRODUCTION}

Urban sensor networks covers a large number of applications, such as water, gas and electricity metering, waste and parking management, air pollution monitoring, public lightning monitoring or self service bike renting. Each of these applications have their own constraints, in terms of duty cycle, periodicity, or emitted power. Short Range Devices (SRD) emitting on the $868 \mathrm{MHz}$ band are usually used with a total potential bandwidth of $8 \mathrm{MHz}$ [1].

These networks are usually composed of sensors that only send data and sink nodes that gather data and transmit them to central database [1]. Several technologies coexist to fulfill each application's constraints [2], using for example spread-spectrum techniques to minimize the interference risk or to allow a better sensitivity [3].

A problem caused by the coexistence of these technologies is that the sinks must be able to receive signals using several technologies. The solution commonly adopted by manufacturers is to have a stack up of receivers dedicated to each technology, and to assemble the data once converted into a digital form. This method has some drawbacks. Firstly, it lacks flexibility: it does not allow reconfiguration when sensors using a new technology are added to the network. Secondly, it is costly: since the global cost of the architecture is almost the sum of individual costs of all individual receivers, some of the receivers' components could be re-used instead of being dedicated to one technology in particular.

This problem can be addressed by designing a reconfigurable gateway. In the ideal case, such a gateway should be able to digitize the whole band immediately after down-conversion to proceed digitally with all the signal processing, as it is done in Software-Designed Radio (SDR). This implies the possibility of receiving signals from various applications and so with different constraints. It is supposed in this paper that no power control is applied to the emitting nodes, so the signals may have very different amplitude levels depending on their location and propagation conditions. This may lead to the well-known near-far problem.

In this paper, the constraints on the Analog-to-Digital Converter (ADC) are firstly evaluated taking into account the particularities of the used technologies. Secondly, the near-far problem effect on digitization is treated, and a method to evaluate the ADC resolution as a function of the received signal powers is proposed.

\section{URBAN SENSOR NETWORK TECHNOLOGIES}

The most representative technologies used in urban sensor networks are selected in this study. The ones provided by the IEEE $802.15 .4 \mathrm{k}$ 's specifications [4] and WMBus are chosen. WMBus is described in the standard in development EN 13757-4 [5].

IEEE $802.15 .4 \mathrm{k}$ has two modes: it can employ a DSSS mode, with a spreading factor up to 32768 , or a FSK mode, using a FSK modulation with a spreading factor limited to 16. WMBus uses a FSK modulation, without spread spectrum. The DSSS technique allows to reach negative Signal-to-Noise Ratios (SNRs) when transmitting data. This is usually not taken into account when estimating the ADC's SNR because there is an hypothesis of a positive SNR. With a negative SNR, the noise is scaled to the ADC's dynamic range, whereas with a positive SNR, it is the signal that is scaled to the dynamic range. This fact justifies a new estimation of the ADC SNR, when DSSS is used, in the context of urban sensor network. 


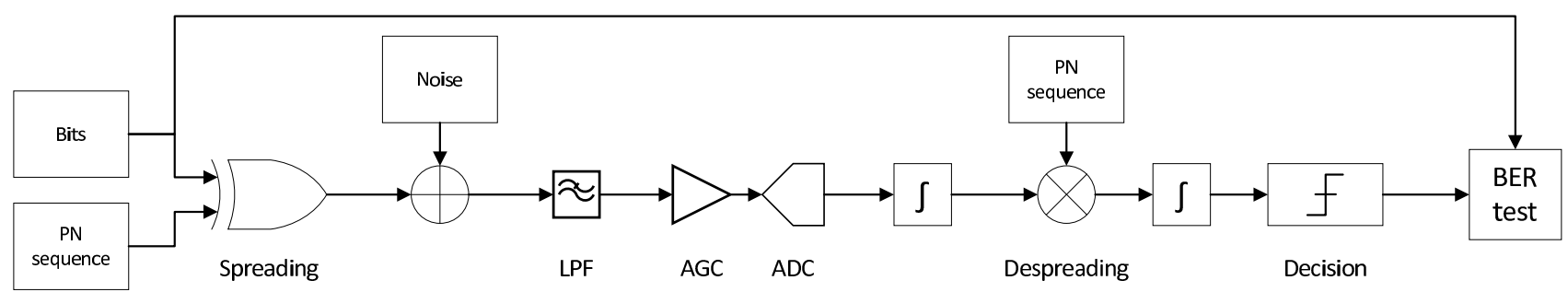

Fig. 1. Simulated transmission chain in Agilent ADS

\section{QUANTIZATION REQUIREMENTS ON A SPREAD SIGNAL}

To find the best resolution when using DSSS (i.e. the smallest resolution that allows to properly demodulate the data), a simulation has been set up on Agilent ADS software[6], returning the Bit Error Rate (BER) for a given SNR and ADC resolution. The simulated transmission chain is shown in Fig. 1. A binary signal is first generated and spread using a pseudo-noise (PN) sequence. Then, a white Gaussian noise is added to the signal, which is filtered, digitized and de-spread. The quality of the transmission is measured via the BER with the Monte Carlo method.

Fig. 2 shows the simulated BER as a function of the ADC resolution. The BER is plotted for several $E_{b} / N_{0}$ to show the ADC's performance in a large enough operating range. It can be seen that BER is degraded with a low $\mathrm{ADC}$ resolution, because quantization noise is stronger with a lower ADC resolution. If ADC resolution increases, quantization noise is smaller, and thermal noise becomes preponderant. $E_{b} / N_{0}$ converges therefore when quantization noise becomes negligible compared to thermal noise. This happens from a resolution of 4 bits, or 5 bits when $E_{b} / N_{0}$ is $9 \mathrm{~dB}$, as it can be seen on Fig. 2. The worst resolution value is kept in the following to guarantee a good digitization in every case.

It is thus considered that a resolution of 5 bits is required to properly demodulate a spread signal. This confirms what is commonly done in DSSS systems, where an empirical value of 5 bits is considered to be necessary for digitizing one signal.

\section{Simultaneous ReCePtion of two Signals}

\section{A. Resolution requirements analysis}

To understand what the near-far problem implies on the receiver architecture, the ADC resolution has to be expressed as a function of the power of two signals of different magnitudes. It is supposed that a high and a low strength signals are simultaneously treated on the receiver in the same frequency band, but in different channels to allow filtering. The RMS magnitude of the strong signal is noted $S_{s}$, and the RMS magnitude of the weak one is noted $S_{w}$. The input signal (whose amplitude is the sum

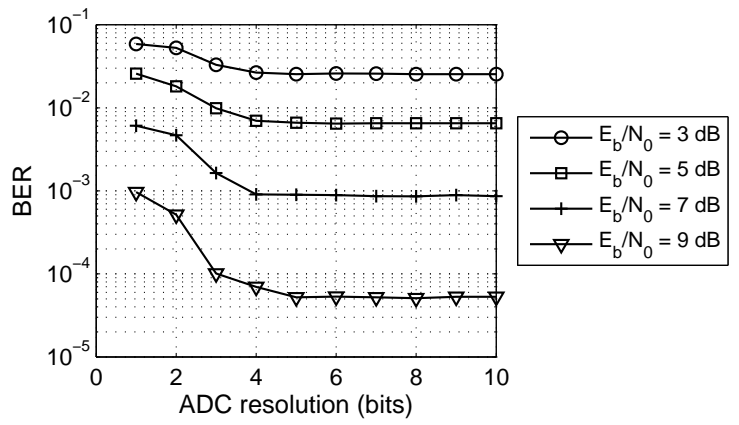

Fig. 2. Simulated BER vs ADC resolution of a DSSS signal with a spreading factor of 16

of the weak and strong signals' amplitudes) is scaled to the dynamic range of the ADC. In this subsection, we find the resolution that introduces the same quantization noise on the weak signal when digitizing simultaneously two signals than if only the weak signal was digitized.

As the dynamic range can be expressed as $\Delta \cdot 2^{n}$, where $\Delta$ is the quantization step, one can write:

$$
\Delta \cdot 2^{n}=\frac{\left(S_{s}+S_{w}\right) \cdot 2 \sqrt{2}}{K}
$$

where $K$ is the scaling factor, meaning that $K=0.8$ if the input signal occupies $80 \%$ of the dynamic range.

The quantization noise brought by the ADC is:

$$
N_{Q}=\frac{\Delta}{2 \sqrt{3}}
$$

From (1) and (2), the signal-to-quantization noise ratio (SNQR) of the weak signal can be expressed as:

$$
S N Q R_{w}=\frac{S_{w}}{N_{Q}}=K \cdot \frac{S_{w}}{S_{w}+S_{s}} \cdot 2^{n} \cdot \sqrt{\frac{3}{2}}
$$

If only the weak signal was received, a resolution $n_{w}$ would be needed to have an acceptable BER. Then the SNQR would be:

$$
S N Q R=2^{n_{w}} \cdot \sqrt{\frac{3}{2}}
$$

To find what resolution allows to have the same SNQR (and so the same digitization quality) on the weak signal 
when digitizing a strong and a weak signal, an identification is done between (3) and (4). The result is given in the following equation:

$$
n=n_{w}+\log _{2} \frac{1+\frac{S_{s}}{S_{w}}}{K}
$$

As the resolution $n_{w}$ is known to be 5 bits from the previous section and $K$ is easily assessable, the needed resolution to digitize a weak and a strong signals has been expressed as a function of these signals' amplitudes ratio. The following subsection first estimates the maximum ratio between $S_{w}$ and $S_{s}$, based on a worst-case scenario. Then the minimum required ADC resolution is discussed, with feasibility assessment elements.

\section{B. Minimum required resolution in a worst-case scenario}

The power of the strongest signal is considered to be the power of a signal emitted from a node located at 10 meters from the receiver. This distance has been chosen from the SmartSantander project [7] for its representativeness of what is encountered in sensor networks. The power received at $10 \mathrm{~m}$ from the emitting node is calculated based on the propagation model Winner+ [8]. The emitted power has been estimated to be $14 \mathrm{dBm}$ from existing systems, and Winner+ gives a $48.5 \mathrm{~dB}$ attenuation at $10 \mathrm{~m}$ in urban area. The maximum power that can be received is thus $-34.5 \mathrm{dBm}$.

To evaluate the minimum power that can be received, link budgets have been done for several technologies that are considered to be common in our application. In particular, we studied WMbus and the IEEE 802.15.4k, to obtain the maximum sensitivity of these technologies, and thus the minimum power that must be properly received. The link budgets lead to a received power ranging from -137.2 to $-34.5 \mathrm{dBm}$, hence a $S_{s} / S_{w}$ ratio varying between 0 and $102.7 \mathrm{~dB}$.

This result is injected in (5) to obtain the Fig. 3, where the needed ADC resolution is plotted as a function of $S_{s} / S_{w}$. Useful resolution has been set to 5 bits, according to the simulation results. It can be seen on the Fig. 3 that when $S_{s} / S_{w}=0 \mathrm{~dB}$, the needed resolution is 7 bits. One supplementary bit is indeed needed to digitize two signals of same amplitude, and the use of a scaling factor makes a second supplementary bit necessary.

The limit power ratio is represented in dashed line, and leads to a minimum required resolution of 23 bits with a power ratio between the two signals of $102.7 \mathrm{~dB}$. It can be seen in [9] that an Effective Number Of Bits (ENOB) of 14 bits can be reached when digitizing with a sampling frequency above $10 \mathrm{MS} / \mathrm{s}$. As the sampling frequency must be at least $16 \mathrm{MS} / \mathrm{s}$ to digitize the $8 \mathrm{MHz}$ band, it can be supposed that the ENOB will be lesser than 14 bits.
Other ways must then be found to digitize all the band with a sufficiently high resolution to demodulate simultaneously received signals, like parallel subbands processing.

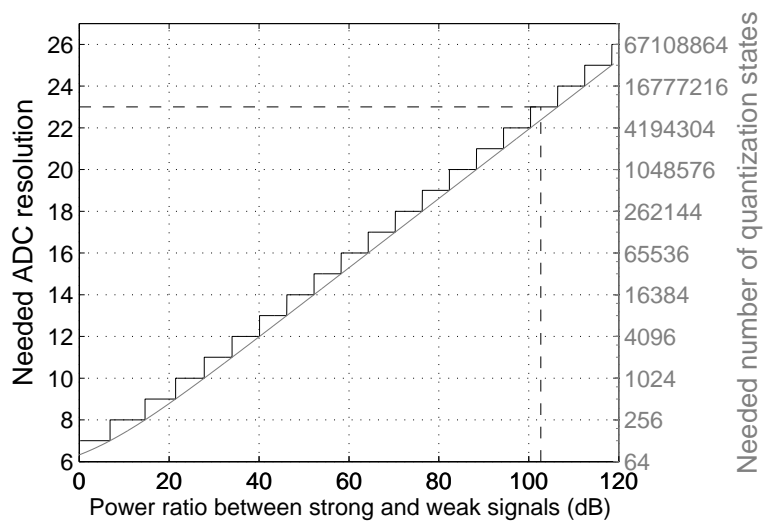

Fig. 3. ADC resolution needed vs power ratio between strong and weak signal

\section{CONCLUSION AND PROSPECTS}

The needed resolution to quantify a spread signal has been evaluated to be 5 bits from simulations on Agilent ADS. Through theoretical calculation, this resolution leads to a needed resolution of 23 bits when digitizing two signals in presence of the near-far problem. This resolution is too big to allow a direct digitization of the $8 \mathrm{MHz}$ band, hence another method must be chosen to digitize all the band.

Several solutions exist: the band can be split in several sub-bands individually digitized, and the gateway's good working can be only guaranteed for a given $S_{s} / S_{w}$ ratio, which must be big enough to cover most of the cases encountered in urban sensor networks.

Oversampling is another way to explore, since one bit of resolution can be added to the ADC resolution by multiplying the sampling frequency by a factor of 4 .

\section{REFERENCES}

[1] ETSI, TR 103 055, september 2011.

[2] L. Mainetti, L. Patrono, and A. Vilei, "Evolution of wireless sensor networks towards the internet of things: A survey," in Software, Telecommunications and Computer Networks (SoftCOM), 2011 19th International Conference on, 2011, pp. 1-6.

[3] H. Lehpamer, RFID Design Principles. Norwood, MA, USA: Artech House, Inc., 2007.

[4] IEEE Std 802.15.4k-2012 (Draft, last revision: october 2012), 2012.

[5] European Standard Std. prEN 13757-4, Rev. 2011: E, September 2011, draft.

[6] Agilent Avanced Design System, http://www.home.agilent.com/en/pc-1297113/advanced-designsystem-ads.

[7] Smart Santander, http://smartsantander.eu/.

[8] J. Meinilä et al., WINNER+ Final Channel Models, Petteri Heino ed., Wireless World Initiative New Radio - WINNER+, June 2010, delivrable D5.3.

[9] B. Jonsson, "A survey of A/D-Converter performance evolution," in Electronics, Circuits, and Systems (ICECS), 2010 17th IEEE International Conference on, 2010, pp. 766-769. 